\title{
A hybrid finite element - statistical energy analysis approach to robust sound transmission modelling
}

\author{
Edwin Reynders ${ }^{\mathrm{a}, \mathrm{b}, *}$, Robin S. Langley ${ }^{\mathrm{b}}$, Arne Dijckmans ${ }^{\mathrm{a}}$, Gerrit Vermeir ${ }^{\mathrm{a}}$ \\ ${ }^{a}$ University of Leuven (KU Leuven), Department of Civil Engineering, Kasteelpark Arenberg 40, B-3001 Leuven, Belgium \\ ${ }^{b}$ University of Cambridge, Department of Engineering, Trumpington Street, Cambridge CB21PZ, UK
}

\begin{abstract}
When considering the sound transmission through a wall in between two rooms, in an important part of the audio frequency range the local response of the rooms is highly sensitive to uncertainty in spatial variations in geometry, material properties and boundary conditions, which have a wave scattering effect, while the local response of the wall is rather insensitive to such uncertainty. For this mid-frequency range, a computationally efficient modeling strategy is adopted that accounts for this uncertainty. The partitioning wall is modeled deterministically, e.g. with finite elements. The rooms are modeled in a very efficient, nonparametric stochastic way, as in statistical energy analysis. All components are coupled by means of a rigorous power balance. This hybrid strategy is extended so that the mean and variance of the sound transmission loss can be computed as well as the transition frequency that loosely marks the boundary between low- and high-frequency behavior of a vibro-acoustic component. The method is first validated in a simulation study, and then applied for predicting the airborne sound insulation of a series of partition walls of increasing complexity: a thin plastic plate, a wall consisting of gypsum blocks, a thicker masonry wall and a double glazing. It is found that the uncertainty caused by random scattering is important except at very high frequencies, where the modal overlap of the rooms is very high. The results are compared with laboratory measurements, and both are found to agree to within the prediction uncertainty in the considered frequency range.
\end{abstract}

Keywords: Building acoustics, mid-frequency methods, uncertainty quantification, airborne sound insulation

\section{Introduction}

The airborne sound insulation of a partitioning structure does not only depend on the material properties and the dimensions of the partition itself, but also on those of the acoustic spaces that it connects, on the boundary conditions, and on the measurement setup. This introduces uncertainty in the acoustic performance of the partition in a specific design situation, because the configuration and occupation of a particular room are generally unknown in advance, and manufactured components are employed in multiple different situations. Even in standardized laboratory conditions, the measured sound transmission loss (also called sound reduction index) can differ significantly from one laboratory to another or for different measurement setups within the same laboratory $[1,2,3,4,5,6]$.

As a result there has been much practical interest in quantifying the uncertainty of sound insulation values; see, e.g., [7] and the references therein. A robust model-based prediction should account for the uncertainty caused by a lack of precise information that would enable to compute exact results, given a correct model type, and errors that are due to the limited predictive performance of a certain model type. In this paper, it is assumed that a model type of sufficient predictive performance is available, so that the prediction errors are dominated by a lack of precise information. A distinction is made between (i) uncertainty in nominal, gross (acoustic) mass and stiffness parameters and damping, and (ii) uncertainty in local (acoustic) mass and stiffness variations, which have a scattering effect. When considering the rooms for example, gross mass and stiffness parameters are the overall sound speed, air density, total room volume and total boundary surface, while uncertainty in the local acoustic mass and stiffness is caused by

\footnotetext{
*Corresponding author. Email: edwin.reynders@bwk.kuleuven.be. Tel.: +3216321677. Fax: +3216321988. Postprint submitted to Journal of Sound and Vibration

Published version: E. Reynders, R.S. Langley, A. Dijckmans, and G. Vermeir. A hybrid finite element - statistical energy analysis approach to robust sound transmission modelling. Journal of Sound and Vibration, 333(19):4621-4636, 2014. http://dx.doi.org/10.1016/j.jsv.2014.04.051
} 


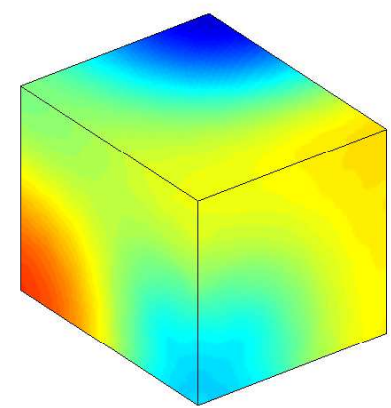

(a)

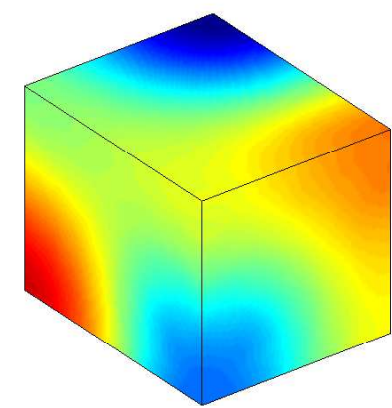

(b)

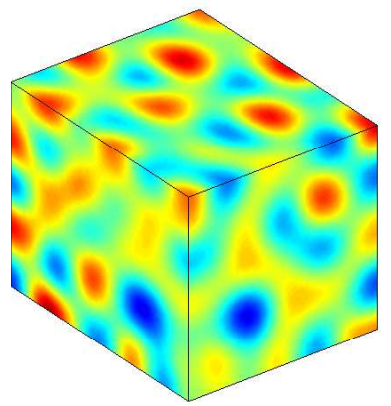

(c)

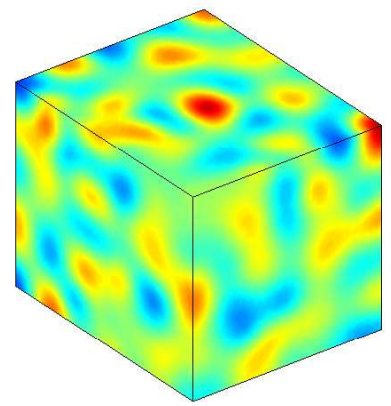

(d)

Figure 1: Sound pressure on the surfaces of a hard-walled room of $4.15 \mathrm{~m} \times 5.09 \mathrm{~m} \times 4.12 \mathrm{~m}$ and with a reverberation time of $1.5 \mathrm{~s}$, excited by a harmonic volume acceleration source of amplitude $1 \mathrm{~m}^{3} / \mathrm{s}^{2}$ at location $(1.2 \mathrm{~m}, 1.2 \mathrm{~m}, 1.2 \mathrm{~m}$ ), measured from one of the room's corners. (a) uniform room, $50 \mathrm{~Hz}$. (b) perturbed room, $50 \mathrm{~Hz}$. (c) uniform room, $250 \mathrm{~Hz}$. (d) perturbed room, $250 \mathrm{~Hz}$. The perturbed room contains 100 added acoustic masses, each having $0.05 \%$ of the nominal acoustic mass $V / c^{2}$ of the room, with $V$ the room volume and $c=343 \mathrm{~m} / \mathrm{s}$ the sound speed. The sound pressure ranges from $-0.7 \mathrm{~Pa}$ (dark blue) to 0.7 $\mathrm{Pa}$ (dark red) (color online).

a lack of knowledge on geometric details of room boundaries or objects in the room. This article is concerned mainly with the second type of uncertainty, and more precisely, how it can be quantified and exploited to result in a robust and computationally efficient mathematical model for sound insulation assessment.

When a vibro-acoustic component (e.g. a room or wall) is modally sparse, it displays little sensitivity to local mass and stiffness variations, and it is essentially deterministic for fixed gross mass and stiffness parameters and damping. In this low-frequency regime, displacement-based methods can be employed [8, 9, 10, 11], and the effect of uncertainty in gross mass and stiffness parameters and damping can be accounted for e.g. by means of Monte Carlo simulation. At high frequencies, however, this approach leads to a high computational cost as (i) the short wavelength of deformation requires a high number of degrees of freedom [12], and (ii) the possibly high sensitivity to small local mass and stiffness variations requires the consideration of an ensemble of randomized models, even for fixed gross mass and stiffness parameters and damping [13]. This is illustrated in Fig. 1 for a hard-walled room: with increasing frequency, the wavelength becomes smaller and the sensitivity of the local response to small local acoustic mass changes becomes larger. Such changes may for instance be caused by the placement or reconfiguration of objects in the room, such as reflecting panels in a laboratory room or furniture in real rooms.

When the local response of a component is highly sensitive to local variations of mass and stiffness, it can be modeled as a pure-tone diffuse reverberant field, as in Statistical Energy Analysis (SEA). In this approach, the response of each subsystem is characterized with a single random variable: its total energy. The uncertainty of a subsystem to local mass and stiffness variations can be measured with the statistical overlap factor, which equals twice the standard deviation of a natural frequency to this type of uncertainty, divided by the mean natural frequency spacing [14, 15]. This quantity is fundamentally different from the modal overlap factor, which is the product of the damping loss factor and the frequency, divided by the mean natural frequency spacing. The modal overlap is a measure for how many modes contribute to the mean response at a particular frequency, while the statistical overlap is a measure for how well the natural frequencies mix with respect to each other across the random ensemble of rooms. Both descriptors are of high practical importance: the SEA assumptions are typically valid when the statistical overlap is larger than unity, while the modal overlap has an important influence on the accuracy of SEA predictions in the sense of dispersion of results around the mean values [16, Sec. 4.2.2].

Until recently, only the mean energy values could be reliably estimated in an SEA analysis [17, 18, 19]. Useful information could therefore be obtained only at high modal overlap (i.e., high modal density and/or damping), where the probability of the total vibro-acoustic energy of the subsystem is narrowly distributed around the mean value. For the computation of the response variance, an extension of the diffuse field model is necessary because the secondorder statistics of the natural frequency spacings in high-frequency regime are needed. Weaver [20] found that, for the generic case where the considered spatial uncertainty does not preserve subsystem symmetries, the statistics of the local eigenvalue spacings saturate to those of the Gaussian Orthogonal Ensemble (GOE) matrix from random matrix theory [21], rather than to the other probability distributions that had been proposed earlier [22, 23]. This universal 
result holds irrespectively of the underlying uncertain parameters and their detailed statistics, and thus allows for a nonparametric description of the uncertainty: only the modal density, the total mass and the damping loss factor of a subsystem are needed in order to predict not only the mean but also the variance of its total energy [24, 25]. Under additional assumptions such as weak coupling, the expressions for an isolated subsystem can be generalized to a built-up system in an elegant way, resulting in an effective SEA variance theory [26, 27]. Recently, also the marginal probability density function of the total energy has been derived for some special but important cases [28, 29]. These results are valid when the damping and gross mass and stiffness parameters are deterministic; if this is not the case, the effect of uncertain parameters can be included by applying the rules of conditional probability.

When some but not all parts of the overal vibro-acoustic system are sensitive to small imperfections, neither the low- or high-frequency approaches discussed above are adequate. However, a hybridization of both is possible, thanks to a reciprocity relationship that links the mean vibrational energy of an SEA subsystem to the variance of the nodal forces at its boundary [30, 15]. Expressions for the mean [31], variance [32] and probability distribution function [28] of the vibrational energies in the SEA subsystems, and of the squared response amplitudes of the deterministic and interface degrees of freedom (DOFs), have been derived. They only account for uncertainty in local variations of mass and stiffness, but they are easily generalized to include other types of uncertainty by applying the rules of conditional probability, as in pure SEA modeling [33].

The emphasis in this paper is on sound transmission loss prediction in the mid-frequency range, which is defined here as the frequency regime where the rooms can be modeled as nonparametric random subsystems while the wall cannot. This range is large when a stiff (i.e., modally sparse) wall is placed in between large (i.e., modally dense) rooms. The hybrid mid-frequency method for vibro-acoustic analysis is extended and applied to the particular problem of predicting the sound transmission loss of a partitioning structure in between two rooms. The extensions allow computing (i) the transition frequency that loosely marks the boundary between low- and high-frequency behavior of a vibro-acoustic component, and (ii) the mean and variance of the sound transmission loss. The applications cover the sound transmission loss prediction of wall structures of increasing complexity: a thin polymethyl metacrylate (PMMA) plate, a gypsum block wall, a thick perforated brick wall, and a double glazing. The considered vibroacoustic system is naturally decomposed into three parts: the emitting room (subsystem 1), the partitioning structure (subsystem 2), and the receiving room (subsystem 3). In the considered frequency range, the rooms (subsystems 1 and 3) can be modeled as SEA subsystems while the partition wall (subsystem 2) cannot.

It may be noted that coupling detailed deterministic wall models to diffuse room models is a common strategy for deterministic sound transmission loss prediction; see, e.g., [34, 35, 36, 37, 38] for recent applications. The expected value of the total transmission loss is then computed by integrating the plane-wave transmission loss over all angles of incidence, which can be computationally costly if numerical integration is needed. The approach presented here is not only more computationally efficient, it is also more general and more robust. This is because sound-structure interaction is accounted for by means of the diffuse field reciprocity relationship, and because not only the expected (mean) transmission loss, but also its variance can be computed thanks to the hybrid variance theory.

\section{The hybrid FE-SEA modeling approach}

As discussed previously, the transmission suite is divided into three components (room - partition wall - room). In order to explain how a component with large statistical overlap can be modelled in an efficient, nonparametric stochastic way, and how it can be coupled to other components that do not necessarily have large statistical overlap, the existing hybrid finite element - statistical energy (FE-SEA) method is concisely summarized in sections 2.1 to 2.3. Subsequently, two new results are presented: the associated standard deviation of the sound transmission loss is derived in section 2.4, and an extensive numerical validation is performed in section 2.5 .

\subsection{Notations and definitions}

In this section, the basic concepts and terminology of the hybrid method are presented as they were introduced in [31]. The deterministic components of the transmission suite and the SEA subsystem interfaces are termed the master system. The response DOFs at circular frequency $\omega$ of the master system are collected in an amplitude vector $\mathbf{q} \in$ $\mathbb{C}^{N_{\text {dof }}}$, so that the time-domain response of the master system is given by $\operatorname{Re}\left(\mathbf{q} \mathrm{e}^{i \omega t}\right)$. Similarly, the external harmonic 
loads at frequency $\omega$ applied at these degrees of freedom are collected in the load amplitude vector $\mathbf{f} \in \mathbb{C}^{N_{\text {dof }}}$. The equations of motion of the whole system are then

$$
\mathrm{Dq}=\mathbf{f}
$$

where $\mathbf{D} \in \mathbb{C}^{N_{\text {dof }} \times N_{\text {dof }}}$ denotes the dynamic stiffness matrix at frequency $\omega$. D is a random matrix because it represents the dynamic behavior of the overall vibro-acoustic system, including the SEA subsystems. It may be decomposed as the sum of the dynamic stiffness matrix of the master system, denoted as $\mathbf{D}_{\mathrm{d}}$, and the dynamic stiffness matrices of the $N_{r}$ SEA subsystems, denoted as $\mathbf{D}_{k}, k=1, \ldots, N_{r}$ :

$$
\mathbf{D}=\mathbf{D}_{\mathrm{d}}+\sum_{k=1}^{N_{r}} \mathbf{D}_{k}
$$

The dynamic stiffness matrix of an SEA subsystem is decomposed as

$$
\mathbf{D}_{k}=\mathbf{D}_{\text {dir }}^{(k)}+\mathbf{D}_{\text {ran }}^{(k)}
$$

where $\mathbf{D}_{\text {dir }}^{(k)}$ denotes the mean of the subsystem's dynamic stiffness matrix: $\mathbf{D}_{\text {dir }}^{(k)}:=\mathrm{E}\left[\mathbf{D}_{k}\right]$. With this decomposition, the equations of motion for an SEA subsystem can be written as

$$
\mathbf{D}_{\text {dir }}^{(k)} \mathbf{q}=\mathbf{f}_{k}+\mathbf{f}_{\text {ran }}^{(k)}
$$

where the reverberant forces are defined as $\mathbf{f}_{\text {ran }}^{(k)}:=-\mathbf{D}_{\text {ran }}^{(k)} \mathbf{q}$, and $\mathbf{f}_{k}$ denotes the sum of the loads applied to subsystem $k$ at its DOFs. The overall equations of motion (1) become

$$
\mathbf{D}_{\text {tot }} \mathbf{q}=\mathbf{f}+\sum_{k=1}^{N_{r}} \mathbf{f}_{\text {ran }}^{(k)}
$$

where $\mathbf{D}_{\text {tot }}:=\mathbf{D}_{\mathrm{d}}+\sum_{k=1}^{N_{r}} \mathbf{D}_{\text {dir }}^{(k)}$ is purely deterministic because it is the mean of the dynamic stiffness matrix of the overall system: $\mathbf{D}_{\text {tot }}=\mathrm{E}[\mathbf{D}]$. The subscript "tot" emphasizes that $\mathbf{D}_{\text {tot }}$ is the total deterministic part of $\mathbf{D}$.

\subsection{Mean harmonic response}

When the statistical overlap in all SEA subsystems is large enough and the random properties of different subsystems are statistically independent of each other, the mean total energy $\hat{E}_{j}$ of subsystem $j$ can be obtained from a stationary power balance which involves the other random subsystems as well as the deterministic master system [31]. For the case where the external loading acts solely on the subsystems, this reads:

$$
\omega\left(\eta_{j}+\eta_{\mathrm{d}, j}\right) \hat{E}_{j}+\sum_{k=1}^{N_{r}} \omega \eta_{j k} n_{j}\left(\frac{\hat{E}_{j}}{n_{j}}-\frac{\hat{E}_{k}}{n_{k}}\right)=\mathrm{E}\left[P_{j}\right], \quad j=1, \ldots, N_{r} .
$$

In this expression, $\eta_{j}$ is the damping loss factor of subsystem $j, n_{j}$ its modal density, $P_{j}$ the power input from external forces applied directly to this subsystem, and

$$
\begin{aligned}
\omega \eta_{\mathrm{d}, j} & =\frac{2}{\pi n_{j}} \sum_{r, s} \operatorname{Im}\left(D_{\mathrm{d}, r s}\right)\left(\mathbf{D}_{\mathrm{tot}}^{-1} \operatorname{Im}\left(\mathbf{D}_{\mathrm{dir}}^{(j)}\right) \mathbf{D}_{\mathrm{tot}}^{-H}\right)_{r s} \\
\omega \eta_{j k} n_{j} & =\frac{2}{\pi} \sum_{r, s} \operatorname{Im}\left(D_{\mathrm{dir}, r s}^{(j)}\right)\left(\mathbf{D}_{\mathrm{tot}}^{-1} \operatorname{Im}\left(\mathbf{D}_{\mathrm{dir}}^{(k)}\right) \mathbf{D}_{\mathrm{tot}}^{-H}\right)_{r s}
\end{aligned}
$$

where the superscript ${ }^{H}$ denotes Hermitian transpose. If the subsystems are weakly coupled through the master system, $\mathrm{E}\left[P_{j}\right]$ can be approximated as the power input to the mean uncoupled subsystem. It can be noted that the power balance equation (6) has the same structure as in conventional SEA. Therefore the factors $\eta_{j k}$ represent coupling loss 
factors, and (8) provides a rigorous and straightforward way to compute them, even in the mid-frequency range where the overall vibro-acoustic system contains purely deterministic components. The coupling loss factors, as computed from (8), automatically contain the effects of both resonant and non-resonant transmission, while in conventional SEA, these effects need to be considered separately [39].

Still under the assumptions that the statistical overlap in all SEA subsystems is large enough and that the random properties of different subsystems are independent of each other, the frequency-dependent matrix $\mathbf{D}_{\text {dir }}^{(k)}$ can be computed as the direct field receptance matrix of subsystem $k[30,39]$. The term 'direct field' denotes the part of the subsystem response containing incoming waves only; it is the limiting response that would be observed at the interface when the extent of the subsystem would be increased towards infinity. For the case of a transmission suite, where two rooms are separated by a plane flexible structure of finite size, the direct field dynamic stiffness matrix of a room as seen by the structure corresponds to the one of a grid of points covering the interface between the room and the structure, but embedded in an infinite planar baffle. The related Rayleigh integral may be numerically evaluated based on, for instance, a Fourier transform [40] or wavelet approach [41]. In this work the wavelet approach is adopted.

\subsection{Variance of the harmonic response}

The matrix representation of (6) is

$$
\mathbf{C}_{\mathbf{0}} \mathrm{E}[\overline{\mathbf{E}}]=\mathrm{E}[\mathbf{P}]
$$

where $\overline{\mathbf{E}}$ denotes the vector of total energies of the SEA subsystems, divided by their modal density: $\bar{E}_{k}:=E_{k} / n_{k}$. A power balance of the form (9) can also be written for each particular realization of the hybrid deterministicnonparametric random system:

$$
\mathbf{C E}=\mathbf{P} \text {. }
$$

A first-order perturbation analysis of this power balance has been performed in [32], resulting in the following expression for the covariance of the subsystem energies:

$$
\begin{aligned}
\operatorname{Cov}\left[\bar{E}_{i}, \bar{E}_{j}\right]= & \sum_{k=1}^{N_{r}} \sum_{s=1}^{N_{r}} C_{0, i k}^{-1} C_{0, j s}^{-1} \operatorname{Cov}\left[P_{k}, P_{s}\right] \\
& +\sum_{k=1}^{N_{r}} \sum_{\substack{p=1 \\
N_{r}}}^{N_{r}} \sum_{\substack{s=1 \\
s \neq k}}^{N_{r}}\left(C_{\substack{r=1 \\
r \neq p}}^{-1}-C_{0, i s}^{-1}\right)\left(C_{0, j p}^{-1}-C_{0, j r}^{-1}\right) \frac{\hat{E}_{s} \hat{E}_{r}}{n_{s} n_{r}} \operatorname{Cov}\left[C_{k s}, C_{p r}\right] .
\end{aligned}
$$

All terms on the right-hand side arise also in the computation of the mean response, except for the covariance terms. As discussed in [26], the statistics of the power inputs $P_{k}$ can be computed from:

$$
\begin{aligned}
\operatorname{Cov}\left[P_{k}, P_{s}\right] & =\delta_{k s} \mathrm{E}\left[P_{k}\right]^{2} r^{2}\left(\alpha_{k}, m_{k}^{\prime}\right) \\
r^{2}(\alpha, m) & =\frac{1}{\pi m}\left(\alpha-1+\frac{1-\mathrm{e}^{-2 \pi m}}{2 \pi m}+\mathrm{E}_{\text {int }}(\pi m)\left(\cosh (\pi m)-\frac{\sinh (\pi m)}{\pi m}\right)\right),
\end{aligned}
$$

where $\mathrm{E}_{\text {int }}$ denotes the exponential integral, and the term $\alpha_{k}$ depends on both the nature of the applied loading and the nature of the mode shapes of subsystem $k$. The term $m_{k}^{\prime}$ in (12) is the effective modal overlap factor of subsystem $k$, which is defined as [17]

$$
m_{k}^{\prime}:=\omega \eta_{k}^{\prime} n_{k}, \quad \text { where } \quad \eta_{k}^{\prime}:=\frac{1}{\omega n_{k} C_{0, k k}^{-1}},
$$

where $\eta_{k}^{\prime}$ is termed the effective loss factor of subsystem $k$. When subsystem $k$ is loaded at a single point and its mode shape at the loading point is, in line with the SEA assumptions, circular complex normally distributed across the ensemble, a value $\alpha_{k}=3$ is expected. This value will be adopted for the random acoustic volumes considered in 
this paper. The relative covariance between two elements of the power balance matrix $\mathbf{C}_{\mathbf{0}}$ is given by [32]

$$
\begin{gathered}
\text { Relcov }\left[C_{k m}, C_{r m}\right]=\sum_{s=1}^{N_{r}} \frac{a_{s}}{\pi m_{s}^{\prime}} \frac{\operatorname{Tr}\left(\tilde{\mathbf{D}}_{\text {dir }}^{(s)} \mathbf{G}^{(m)}\right) \operatorname{Tr}\left(\mathbf{J}_{k s r} \mathbf{G}^{(m)}\right)+\operatorname{Tr}\left(\tilde{\mathbf{D}}_{\text {dir }}^{(s)} \mathbf{G}^{(m)} \mathbf{J}_{k s r} \mathbf{G}^{(m)}\right)}{\operatorname{Tr}\left(\tilde{\mathbf{D}}_{\text {dir }}^{(k)} \mathbf{G}^{(m)}\right) \operatorname{Tr}\left(\tilde{\mathbf{D}}_{\text {dir }}^{(r)} \mathbf{G}^{(m)}\right)} \\
+\frac{\operatorname{Tr}\left(\tilde{\mathbf{D}}_{\text {dir }}^{(k)} \mathbf{G}^{(m)} \tilde{\mathbf{D}}_{\text {dir }}^{(r)} \mathbf{G}^{(m)}\right)}{\operatorname{Tr}\left(\tilde{\mathbf{D}}_{\text {dir }}^{(k)} \mathbf{G}^{(m)}\right) \operatorname{Tr}\left(\tilde{\mathbf{D}}_{\text {dir }}^{(r)} \mathbf{G}^{(m)}\right)}
\end{gathered}
$$

and

$$
\text { Relcov }\left[C_{k m}, C_{r p}\right]=\sum_{s=1}^{N_{s}} \frac{a_{s}}{\pi m_{s}^{\prime}} \frac{\operatorname{Re}\left(\operatorname{Tr}\left(\tilde{\mathbf{D}}_{\text {dir }}^{(s)} \mathbf{G}^{(m)} \mathbf{H}_{k s r} \mathbf{G}^{(p)}\right)\right)}{\operatorname{Tr}\left(\tilde{\mathbf{D}}_{\text {dir }}^{(k)} \mathbf{G}^{(m)}\right) \operatorname{Tr}\left(\tilde{\mathbf{D}}_{\text {dir }}^{(r)} \mathbf{G}^{(p)}\right)}, \quad m \neq p,
$$

where the tilde ( ) is used as an abbreviated notation to represent the imaginary part of a matrix, Tr represents the trace of a matrix, and

$$
\begin{aligned}
\mathbf{G}^{(m)} & :=\frac{4}{\omega \pi} \mathbf{D}_{\text {tot }}^{-1} \tilde{\mathbf{D}}_{\text {dir }}^{(m)} \mathbf{D}_{\text {tot }}^{-H} \\
\mathbf{J}_{k s r} & :=\frac{1}{2}\left(\mathbf{H}_{k s r}+\mathbf{H}_{r s k}\right) \\
\mathbf{H}_{k s r} & :=4 \tilde{\mathbf{D}}_{\text {dir }}^{(k)} \mathbf{D}_{\text {tot }}^{-1} \tilde{\mathbf{D}}_{\text {dir }}^{(s)} \mathbf{D}_{\text {tot }}^{-H} \tilde{\mathbf{D}}_{\text {dir }}^{(r)}+\delta_{k r} \delta_{k s} \tilde{\mathbf{D}}_{\text {dir }}^{(k)}-2 \delta_{r s} i \tilde{\mathbf{D}}_{\text {dir }}^{(k)} \mathbf{D}_{\mathrm{tot}}^{-1} \tilde{\mathbf{D}}_{\text {dir }}^{(r)}+2 \delta_{k s} i \tilde{\mathbf{D}}_{\text {dir }}^{(k)} \mathbf{D}_{\text {tot }}^{-H} \tilde{\mathbf{D}}_{\text {dir }}^{(r)} .
\end{aligned}
$$

The factor $a_{s}$ which appears in (15) and (16) depends on the coupling of subsystem $s$ to the master system. For area couplings as considered in this article, $a_{s}$ will be close to unity; the choice $a_{s}=1$ is therefore adopted throughout this paper.

\subsection{Sound transmission loss}

The airborne sound transmission loss $R$ of a partition wall is defined as the ratio between the incident and transmitted acoustic power [19]. In laboratory conditions, $R$ is determined from the following measurement formula [42]:

$$
R=L_{p 1}-L_{p 3}+10 \log \frac{S_{2}}{A_{3}}
$$

where $L_{p 1}$ and $L_{p 3}$ are the spatially averaged stationary sound pressure levels in the emitting and receiving rooms, respectively, $S_{2}$ is the surface area of the partition and $A_{3}$ the absorption of the receiving room. Eq. (20) can therefore serve as the practical definition of the transmission loss $R$. Both definitions are equivalent, in an ensemble averaged sense, when a diffuse sound field exists in both the emitting and the receiving rooms. The room absorption $A_{3}$ is determined from the reverberation time, the room volume and the sound speed using Sabine's formula.

In stationary conditions, the total energy of a subsystem, consisting of the sum the kinetic and potential energies, is constant. For low damping and at resonance, the kinetic and potential energies at a particular nonzero frequency will be approximately equal [19]. An equivalent expression for (20) is therefore

$$
R=10 \log \frac{E_{1}}{V_{1}}-10 \log \frac{E_{3}}{V_{3}}+10 \log \frac{S_{2}}{A_{3}}=10 \log \frac{E_{1} V_{3} S_{2}}{E_{3} V_{1} A_{3}}
$$

where $E_{1}$ and $E_{3}$ represent the total energy in the emitting and receiving rooms, respectively, and $V_{1}$ and $V_{3}$ their respective volumes. As this expression involves the ratio of the total energies of the rooms, its statistics can only be computed exactly when the joint probability distribution function of the total energies is known. As present this is not the case, and therefore an approximate solution is considered here.

A first-order Taylor expansion of (21) around $R=10 \log \left(\hat{E}_{1} V_{3} S_{2} /\left(\hat{E}_{3} V_{1} A_{3}\right)\right)$ can be analytically computed, resulting in the following approximation for the sound transmission loss:

$$
R \approx 10 \log \frac{\hat{E}_{1} V_{3} S_{2}}{\hat{E}_{3} V_{1} A_{3}}+\frac{10}{\ln (10)}\left[\begin{array}{cc}
\frac{1}{\hat{E}_{1}} & \frac{-1}{\hat{E}_{3}}
\end{array}\right](\mathbf{E}-\hat{\mathbf{E}}) .
$$


With this approximation, the following approximations for the mean of the transmission loss

$$
\mathrm{E}[R] \approx 10 \log \frac{\hat{E}_{1} V_{3} S_{2}}{\hat{E}_{3} V_{1} A_{3}}
$$

and for its variance

$$
\sigma_{R}^{2} \approx \frac{100}{\ln ^{2}(10)}\left(\frac{\operatorname{Var}\left(E_{1}\right)}{\hat{E}_{1}^{2}}-\frac{2}{\hat{E}_{1} \hat{E}_{3}} \operatorname{Cov}\left(E_{1}, E_{3}\right)+\frac{\operatorname{Var}\left(E_{3}\right)}{\hat{E}_{3}^{2}}\right),
$$

are obtained. This equation reveals that the variance of the transmission loss depends, up to first order, on the relative energy covariance only.

Note that the above derivations hold for harmonic quantities (such as energy or sound transmission loss) only, not for band-averaged quantities. While the computation of the mean of band-averaged quantities from mean harmonic quantities is straightforward, this is not the case for the variance. The reason is that for computing the variance of band-averaged quantities, the statistical correlation of these quantities amongst different frequencies is needed, and closed-form expressions for these do not exist at present for the hybrid FE-SEA method.

\subsection{Numerical validation}

In this section the foregoing theory is validated with a simulation example. The aim is to compute the sound transmission loss of a $10 \mathrm{~cm}$ thick gypsum block wall (density $\rho=910 \mathrm{~kg} / \mathrm{m}^{3}$, modulus of elasticity $E=3150 \mathrm{MPa}$, Poisson's ratio $\nu=0.22$, damping loss factor $\eta=0.01$ ), situated in between two rectangular rooms as depicted in Fig. 2. The wall is simply supported. The emitting room is excited by a volume acceleration source, located at $(1.2 \mathrm{~m}, 1.2 \mathrm{~m}, 1.2 \mathrm{~m})$. The nominal sound speed in the rooms is $c=343 \mathrm{~m} / \mathrm{s}$, the air density is $\rho_{\mathrm{a}}=1.20 \mathrm{~kg} / \mathrm{m}^{3}$ and the reverberation time is $T=1.5 \mathrm{~s}$. The relationship between the reverberation time and the damping loss factor of the rooms is

$$
\eta=\frac{4.4 \pi}{\omega T}
$$

The rooms have a random acoustic mass distribution in the sense that a total of $N_{\mathrm{m}}=20$ point air pockets are randomly distributed within each room. Each air pocket has $0.2 \%$ of the total acoustic mass $V / c^{2}$ of the room.

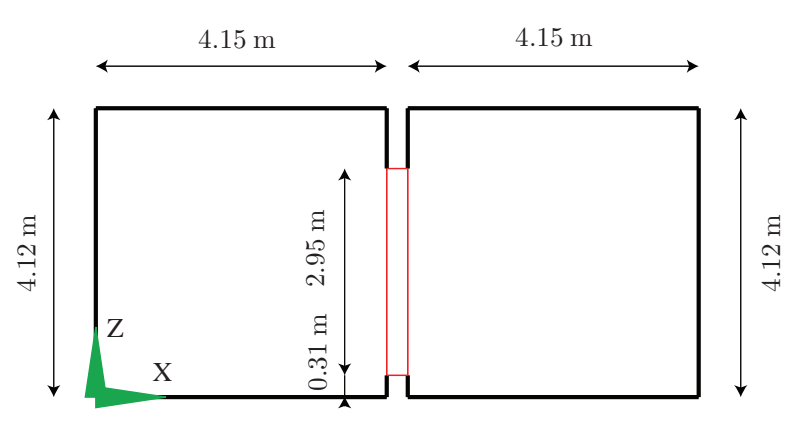

front view

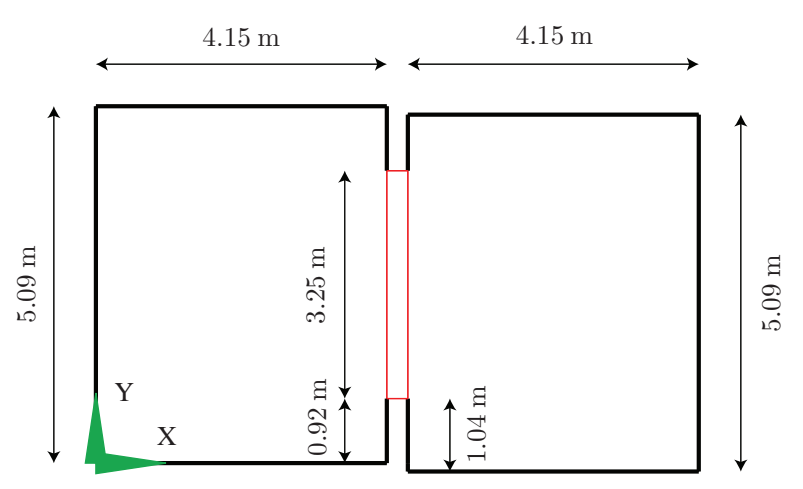

top view

Figure 2: Simulation example: geometry of the room - wall - room system.

Two stochastic models for the transmission suite are constructed. Both models are then compared in terms of the mean and variance of the predicted total room energies, and the mean and variance of the predicted sound reduction index.

The first stochastic model is a modal interaction model [43, Sec. 7.6], in which the random acoustic mass distribution is modeled in detail by means of Monte Carlo simulation. In this model, the analytic hard-walled modes of the rooms, the point air pockets and the analytic modes of the wall are coupled with a Lagrange-Rayleigh-Ritz procedure [7, App.]. Each Monte Carlo realization results in the same modal model of the transmission suite, except that 
the positions of the point air pockets within the rooms are randomly varied across the different realizations. A total of 1000 independent Monte Carlo realizations are constructed, resulting in a total of 1000 independent realizations of the rooms' total time-averaged energy and the sound transmission loss of the system, each based on a different configuration of point air pockets. The considered frequency range is $40-400 \mathrm{~Hz}$.

The second stochastic model is a hybrid model, in which the random acoustic mass distribution is modeled implicitly, by taking the rooms to carry a diffuse reverberant wave field. The wall is modeled with four-node thin shell finite elements that consist of a bilinear membrane element and four overlaid discrete Kirchoff triangle plate elements [44]. The rooms are modeled as SEA subsystems; their modal density is estimated according to [17, Eq. 8.3.5]. In the hybrid model, the mean power input to the random emitting room may be approximated by that of a full space, excited by a harmonic volume acceleration source of amplitude $a_{\mathrm{V}}$ [19, Eq. 7.19]:

$$
\mathrm{E}\left[P_{1}\right]=\frac{a_{V} \rho_{\mathrm{a}}}{8 \pi c}
$$

The hybrid model is computationally very efficient when compared to the full modal model: although the highest response frequency considered here is only $400 \mathrm{~Hz}$, the computational effort needed to solve for both the mean and the variance of the transmission loss in the hybrid model is already less than half the effort needed to compute a single realization with the full modal model.

The mean and variance of the total time-averaged energies in the emitting and the receiving rooms, computed with both the hybrid and the full modal model, are plotted in Fig. 3. The peaks in the mean energy and relative variance of the receiving room, computed with the hybrid method, correspond with resonance frequencies of the bare wall. From around $139 \mathrm{~Hz}$ on, the influence of individual room modes on the statistics of the total energy is small. This frequency is the transition frequency, corresponding to unit statistical overlap, that marks the onset of high-frequency behavior of the rooms. A procedure for estimating the statistical overlap is presented in the Appendix. For reference it can be noted that the modal overlap of the rooms is only 0.7 at $139 \mathrm{~Hz}$. At low frequencies, the relative variance of the response is overestimated because the sound fields in the rooms are not sensitive enough to small random scatterers for reaching the saturating, diffuse field conditions.
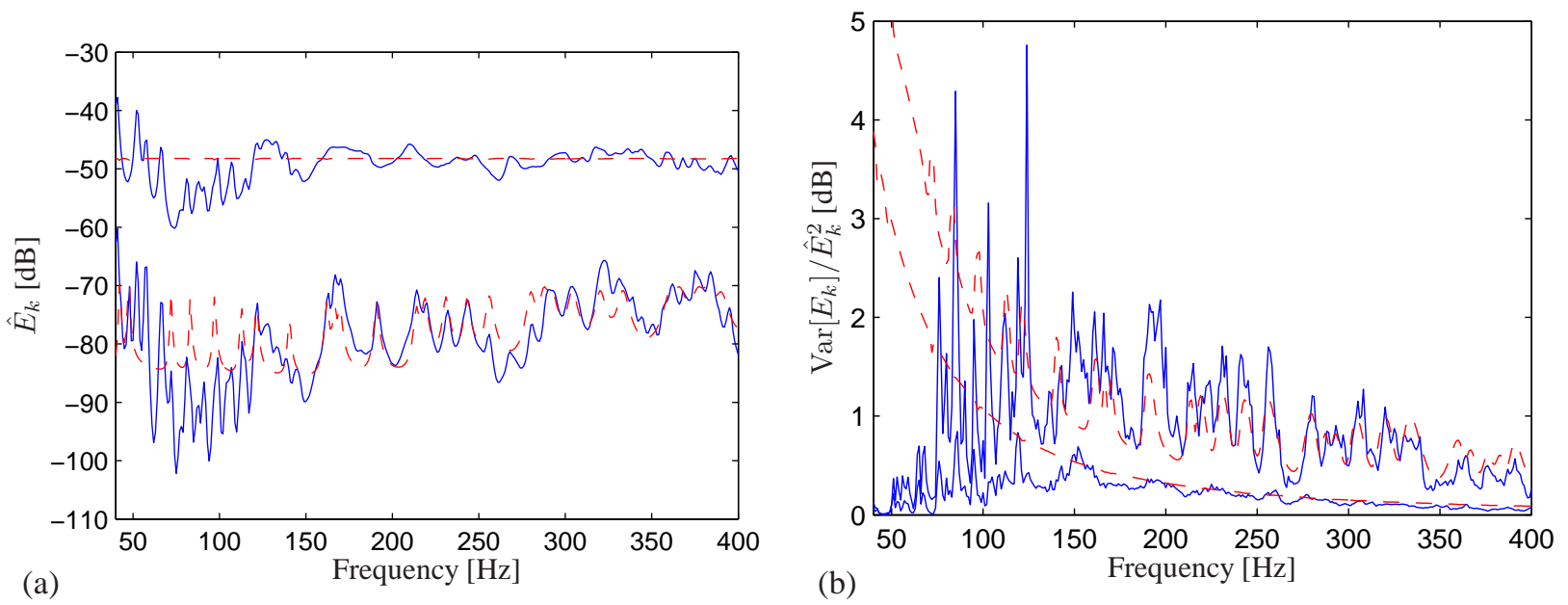

Figure 3: Total energy in the emitting and receiving rooms: (a) mean values and (b) relative variance. Solid lines: Monte Carlo simulation results, obtained with a full modal model. Dashed lines: hybrid FE-SEA model predictions. The smooth curved lines, containing larger mean and smaller relative variance values, correspond to the source room, while the lines with peaks/troughs, containing smaller mean and larger relative variance values, correspond to the receiving room.

The mean and relative variance of the sound transmission loss are shown in Fig. 4. The dips in the transmission loss, computed with the hybrid method, correspond with resonance frequencies of the bare wall. Below the transition frequency of the rooms $(139 \mathrm{~Hz})$, individual resonance modes of the rooms influence the transmission loss statistics, and the relative variance is much smaller than predicted by the hybrid method. At frequencies higher than $139 \mathrm{~Hz}$, 
the assumptions underlying the hybrid room-wall-room model are valid and there is a good agreement between the hybrid model predictions and the computationally much more expensive Monte Carlo simulation results.
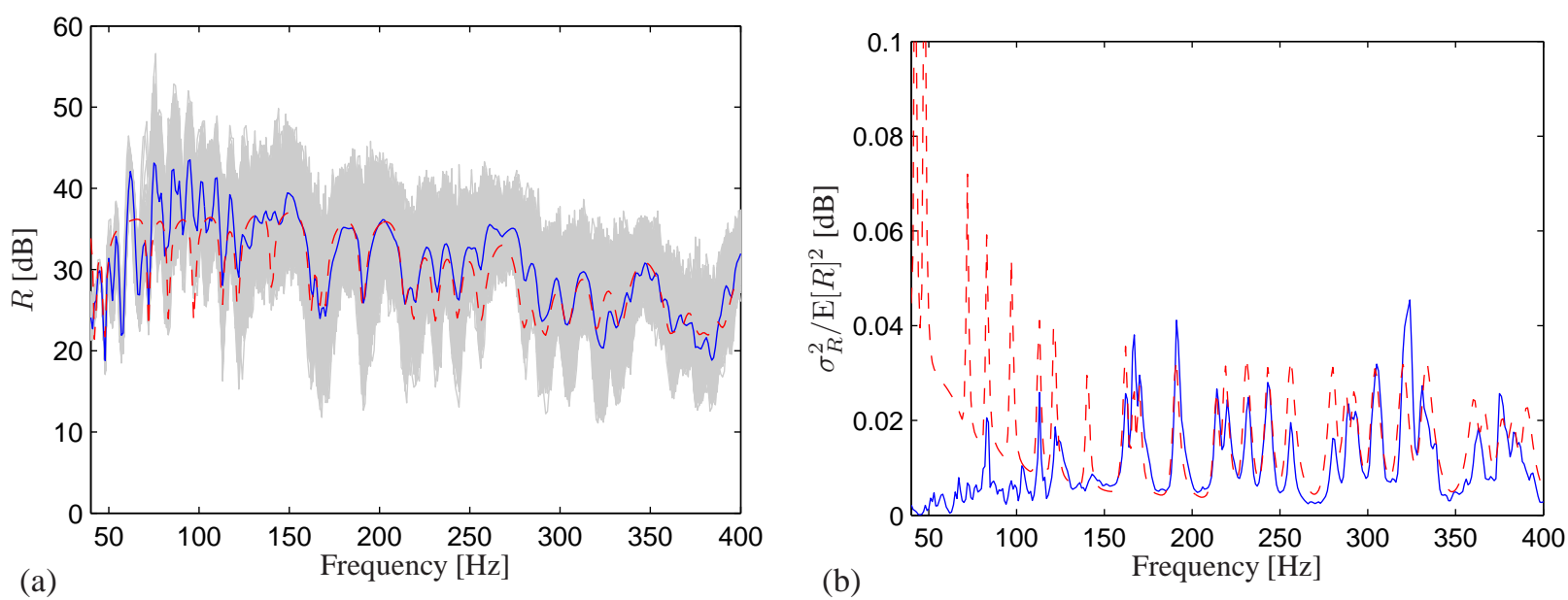

Figure 4: Simulation example, harmonic sound transmission loss: (a) mean and (b) relative variance. Solid line: Monte Carlo simulation. Dotted line: hybrid method. Thin grey lines: individual Monte Carlo realizations.

Besides a validation of the hybrid method against a full modal model, a comparison between the hybrid results and the results of a conventional SEA analysis, which yields mean values only, may also be of interest. A comparison between the mean sound transmission loss of a single wall as computed with conventional SEA and with the more recent hybrid approach has been extensively reported in [39, Sec. 5.1] and it is therefore not repeated here.

\section{Experimental case studies}

In this section, the foregoing methodology is demonstrated and validated by predicting and measuring the sound transmission loss for partition walls in the Laboratory of Acoustics of KU Leuven. Wall systems can be tested in the Laboratory in accordance with the ISO 10140 standard [45] by placing them in a transmission opening between two rooms. The rooms of the Laboratory each have a volume of $87 \mathrm{~m}^{3}$. For the computations, the air density, sound speed and reverberation time of the rooms are taken to be constant with values of $\rho_{\mathrm{a}}=1.20 \mathrm{~kg} / \mathrm{m}^{3}, c=343 \mathrm{~m} / \mathrm{s}$ and $T=1.5 \mathrm{~s}$, respectively.

Four different partition walls are considered: a PMMA panel, a gypsum block wall, a perforated brick wall, and double glazing. In the hybrid prediction models, the single and double walls are part of the deterministic master system, while the rooms are modeled as SEA subsystems. The frequency range in which each hybrid model is valid is therefore the range where the rooms display high-frequency behavior, while the wall still displays low-frequency behavior. Rough estimates of these mid-frequency ranges can be obtained as detailed in the Appendix.

The sound transmission loss of these walls has also been measured in the laboratory. The measurements have been preformed in accordance with the ISO 10140 standard [45], with the exception that the sound pressure levels were averaged over 1/48-octave bands instead of 1/3-octave bands. Three of the tested walls are shown in Fig. 5. For the damping loss factors of the walls, the measured values are used in the computations, unless otherwise specified. These values are listed in Table 1. Note that there is uncertainty in the measured values of the sound insulation, e.g. because the sound pressure levels in the rooms are sampled at a limited number of locations (eight locations were employed in this study). This has been assessed by performing each experiment twice, changing roles between the source and receiving rooms. Although the differences are often significant in the low-frequency range, they are not in the mid-frequency range on which this paper concentrates. The values reported here are the averaged values of both experiments. 


\begin{tabular}{lcccccccccccc}
\hline$f[\mathrm{~Hz}]$ & 125 & 160 & 200 & 250 & 315 & 400 & 500 & 630 & 800 & 1000 & 1250 & 1600 \\
\hline PMMA & 6.6 & 6.4 & 6.0 & 5.9 & 5.8 & 6.0 & 5.7 & 5.7 & 6.1 & 6.2 & 6.5 & 5.6 \\
gypsum wall & 1.9 & 1.9 & 1.4 & 0.9 & 0.6 & 1.1 & 1.2 & 1.3 & 1.4 & 1.4 & 1.4 & 1.1 \\
brick wall & 4.3 & 3.9 & 5.3 & 3.5 & 1.7 & 2.4 & 2.2 & 3.2 & 2.1 & 1.5 & 2.3 & 1.4 \\
glass $6 \mathrm{~mm}$ & 1.9 & 1.7 & 1.8 & 2.2 & 2.2 & 2.6 & 2.8 & 2.7 & 2.0 & 1.6 & 1.7 & 1.6 \\
glass $8 \mathrm{~mm}$ & 2.7 & 2.4 & 2.2 & 2.3 & 2.3 & 3.0 & 2.9 & 2.6 & 2.0 & 1.9 & 1.6 & 1.7 \\
\hline
\end{tabular}

Table 1: Measured damping loss factors $\eta$ (in \%) for the partition walls tested in the Laboratory of Acoustics of KU Leuven.

\subsection{Isotropic PMMA panel}

The first structure is a polymethyl metacrylate panel with density $\rho=1275 \mathrm{~kg} / \mathrm{m}^{3}$, modulus of elasticity $E=$ 4.5 GPa, Poisson's ratio $\nu=0.35$ and dimensions $1.25 \mathrm{~m} \times 1.50 \mathrm{~m} \times 0.015 \mathrm{~m}$. The panel is modeled with thin shell finite elements of the same type as in section 2.5 , and simply supported boundary conditions are assumed. This deterministic model is coupled to the nonparametric stochastic models of the rooms with the hybrid method, and the sound transmission loss is computed.

The mean of the computed sound transmission loss is plotted in Fig. 6 together with the $2 \sigma$ confidence interval (which corresponds to a $95 \%$ confidence interval for a normal distribution). Measured values are also shown. The level of uncertainty in the predictions is striking: the width of the confidence interval decreases slowly from $20 \mathrm{~dB}$ at $139 \mathrm{~Hz}$ to $3 \mathrm{~dB}$ at $1053 \mathrm{~Hz}$, which are the estimated lower and upper bound frequencies, respectively, of the midfrequency range in which the considered hybrid model is valid. In this frequency range, the irregular variations of the measured sound transmission loss are well captured within the predicted confidence interval, although only the uncertainty due to random wave scattering in the rooms is accounted for.

\subsection{Gypsum block wall}

The second structure is a wall of dimensions $3.25 \mathrm{~m} \times 2.95 \mathrm{~m} \times 0.10 \mathrm{~m}$ consisting of gypsum blocks (Fig. 5a). The wall is modeled as isotropic and homogenous, with density $\rho=910 \mathrm{~kg} / \mathrm{m}^{3}$, modulus of elasticity $E=3150 \mathrm{MPa}$, and Poisson's ratio $\nu=0.22$.

The mean and $2 \sigma$ confidence interval of the sound transmission loss are plotted in Fig. 7 for two cases, where the wall is modeled with (a) a thin shell finite element model and simply supported boundary conditions, and (b) a volume

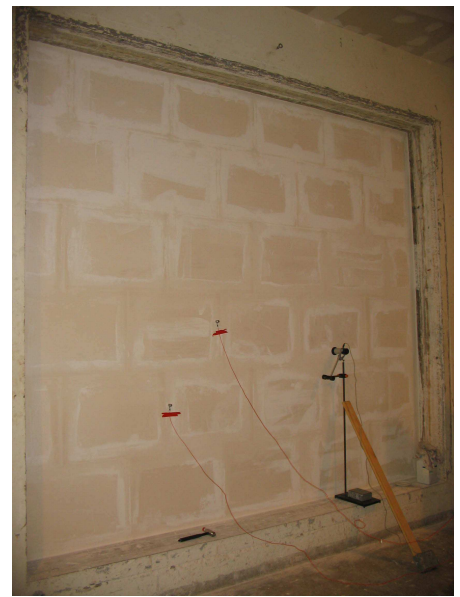

(a)

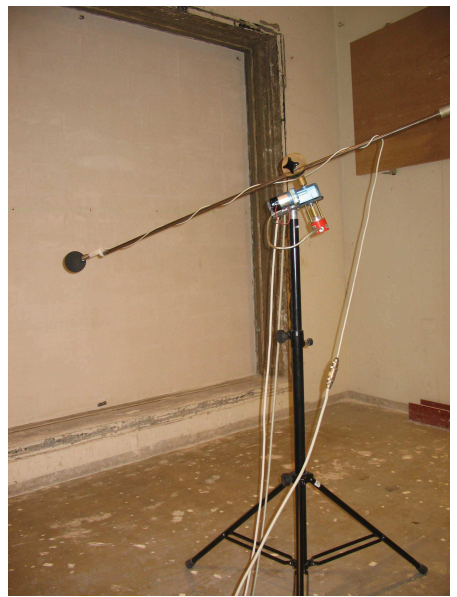

(b)

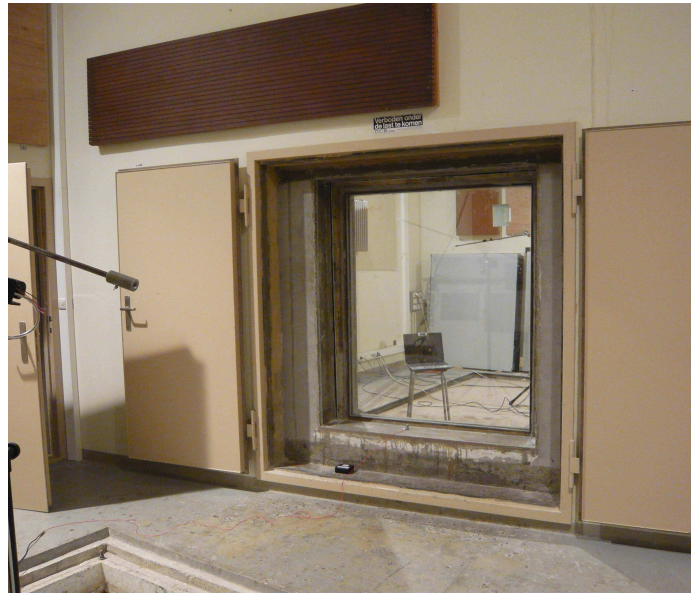

(c)

Figure 5: Partition walls tested in the Laboratory of Acoustics of KU Leuven: (a) gypsum block wall, (b) perforated brick wall, plastered at both sides, and (c) double glazing $6-12-8 \mathrm{~mm}$. 


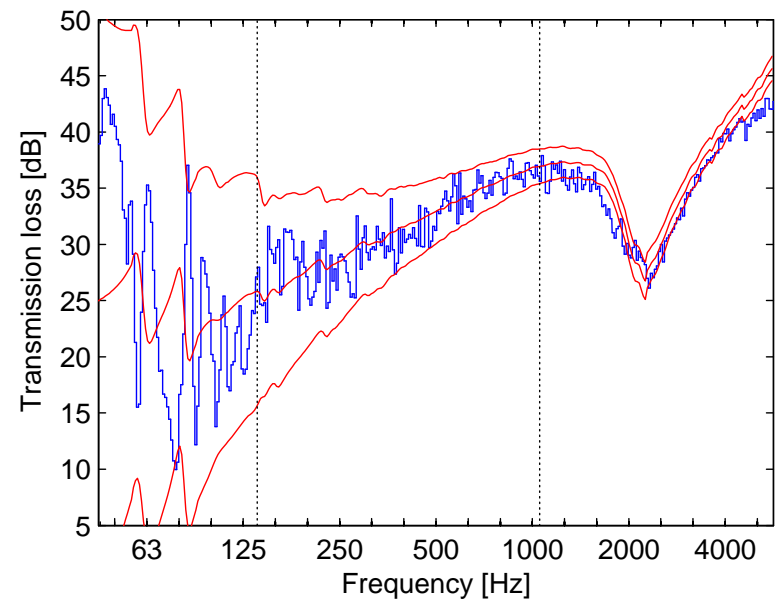

Figure 6: Sound transmission loss of an isotropic PMMA panel. Predicted mean and $2 \sigma$ confidence interval (red) vs. laboratory measurements (blue) (color online). The mid-frequency range is bounded with dotted vertical lines.

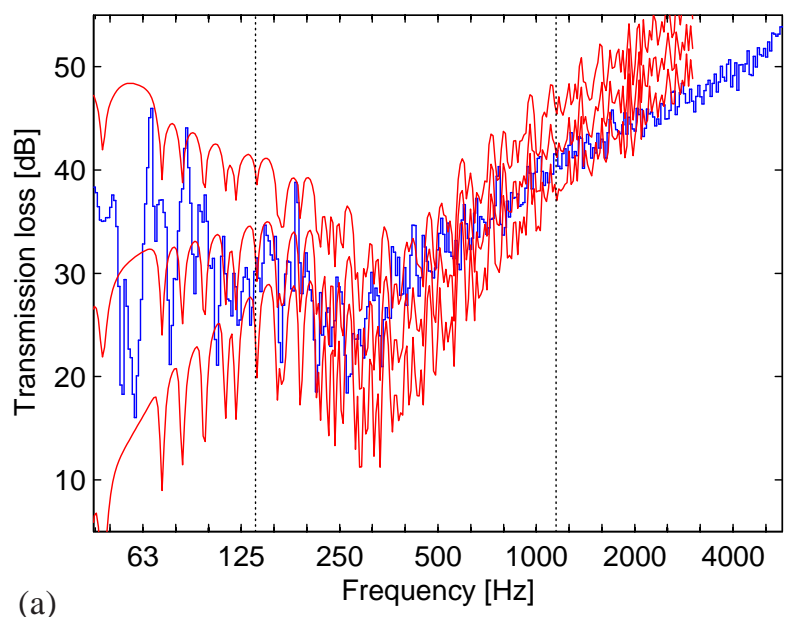

(a)

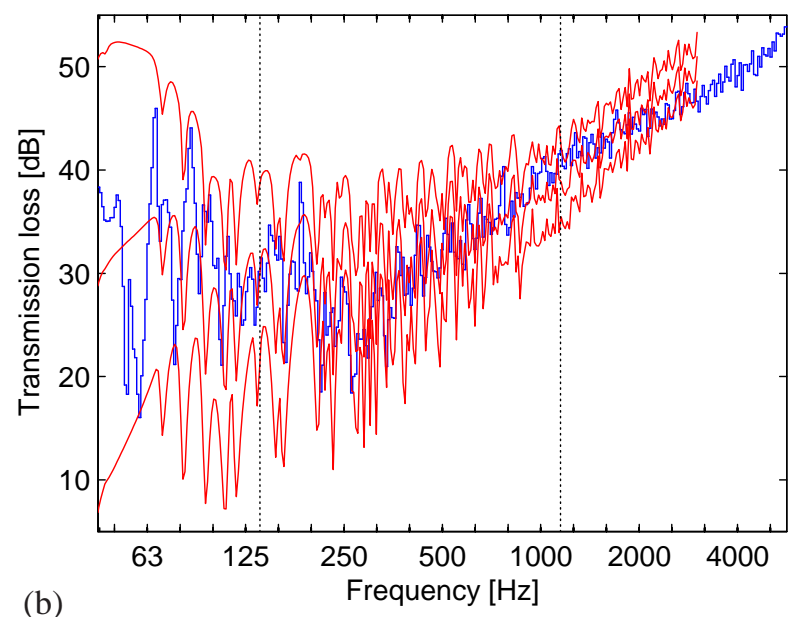

(b)

Figure 7: Sound transmission loss of a gypsum block wall. Predicted mean and $2 \sigma$ confidence interval, computed with a (a) thin shell or (b) volume finite element wall model (red) vs. laboratory measurements (blue) (color online). The mid-frequency range is bounded with vertical lines. 
finite element model, where the boundary displacements in the middle plane are restrained. The thin shell model is constructed using elements of the same type as discussed in section 2.5 , while the volume finite element model is constructed using 8-node linear elastic elements (of the SOLID45 element type in ANSYS). The differences between both results are relatively small in the mid-frequency range. At high frequencies, the plate model overestimates the sound insulation, while the volume model performs better. The width of the confidence interval decreases slowly from $16 \mathrm{~dB}$ at $139 \mathrm{~Hz}$ to $10 \mathrm{~dB}$ at $1149 \mathrm{~Hz}$. The dips in the transmission loss prediction due to modal resonances of the wall are clearly visible, especially at lower frequencies. This is not surprising given the low modal density of the plate which is about $0.09 \mathrm{~Hz}^{-1}$. The dips are also more pronounced than for the PMMA panel. This is due to the smaller damping of the wall: the damping loss factors are 3 to 10 times lower, depending on the frequency (see Table 1). Measured values, averaged over narrow 1/48-octave bands, are also plotted. The uncertainty accounted for in the model can for a large part explain the discrepancies between measurements and model predictions in the mid-frequency range.

\subsection{Lightweight perforated brick wall}

The third structure is a perforated brick wall, plastered at both sides, of dimensions $3.25 \mathrm{~m} \times 1.95 \mathrm{~m} \times 0.19 \mathrm{~m}$ (Fig. 5b). The acoustic behavior of perforated brick walls is complex, given the inhomogeneities at three different scales: that of the fire clay material, that of the brick where small cavities are present in the fire clay because of the perforations, and that of the whole wall where the bricks are held together by mortar layers. When the inhomogeneities are small compared to the wavelength, and when the stiffness is only slightly different in both directions, the wall can be modeled as homogeneous and isotropic. The thickness effects, however, can not be neglected: not only is shear deformation important, thickness resonances (i.e., Lamb modes) are often observed as well in the audio frequency range [46]. Therefore, a volume finite element model of the wall is constructed here, using 8-node solid linear elastic elements (of the SOLID45 element type in ANSYS). The boundary displacements in the middle plane are restrained. Following [11], the equivalent Young's modulus, Poisson's ratio, density and thickness are taken to be $E=1825 \mathrm{MPa}, \nu=0.2, \rho=613.5 \mathrm{~kg} / \mathrm{m}^{3}$ and $t=0.2934 \mathrm{~m}$, respectively.
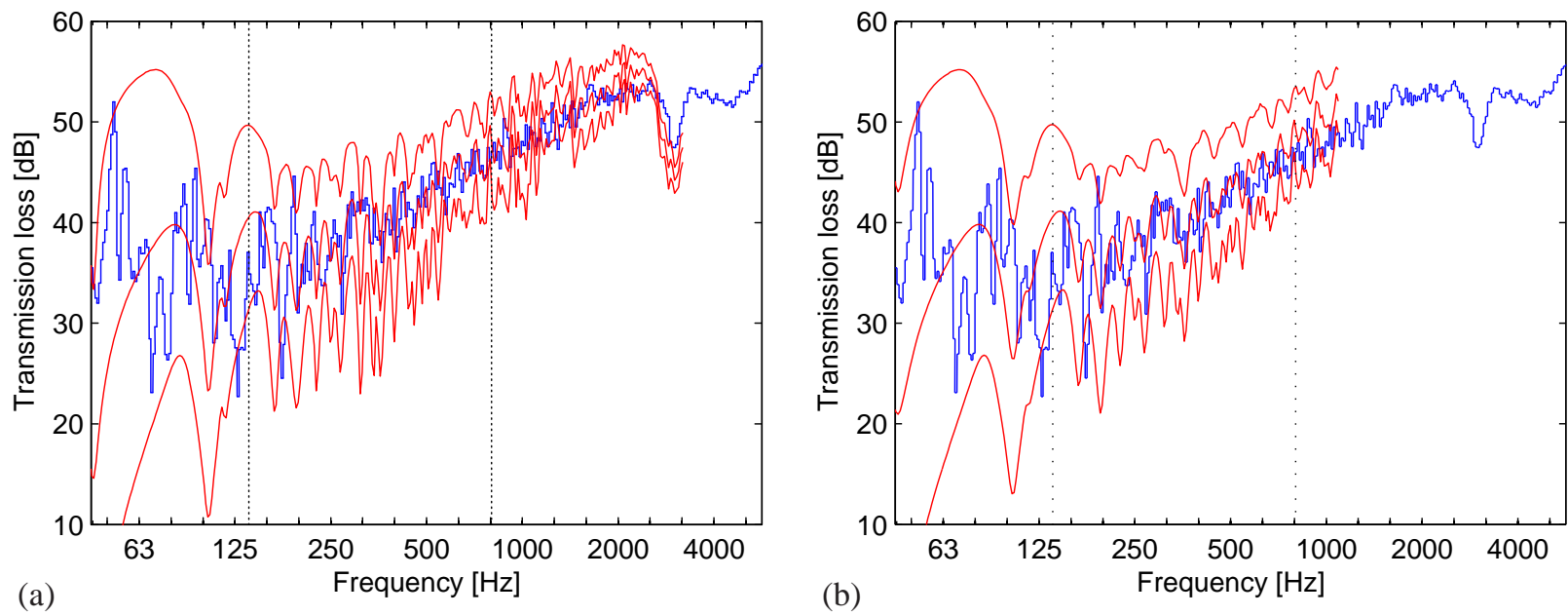

Figure 8: Sound transmission loss of a lightweight perforated brick wall. Predicted mean and $2 \sigma$ confidence interval, computed with the hybrid method (red) when (a) only nonparametric uncertainty is considered, and (b) when additionally the wall damping loss factor $\eta_{2}$ is uniformly distributed according to (28). Laboratory measurements are shown in blue (color online). The mid-frequency range is bounded with vertical lines.

The sound transmission loss predicted with the hybrid method (mean and $2 \sigma$ confidence interval), is plotted in Fig. 8 a together with the measured values which are averaged over 1/48-octave bands. Individual resonance dips of the wall are very clearly visible in the predictions due to the high wall stiffness. A clear dip is also visible around $3000 \mathrm{~Hz}$, caused by the first thickness resonance. The width of the confidence interval in the mid-frequency range decreases slowly from $18 \mathrm{~dB}$ at $139 \mathrm{~Hz}$ to $7 \mathrm{~dB}$ at $801 \mathrm{~Hz}$. In this frequency range, the uncertainty accounted for in the model can for a large part explain the discrepancies between measurements and model predictions. 
In order to illustrate the influence of additional parametric uncertainty, the computation is repeated but with the damping loss factor of the wall $\eta_{2}$ as an additional random variable. This case is often encountered in practice when the transmission loss of a wall is predicted before measurements are carried out. Even if the damping loss factor is derived from measurements, the obtained values have often a large relative error. Based on field measurements, Craik [47] proposed the following estimate for masonry/concrete walls and floors:

$$
\eta_{2} \approx \frac{2.5}{\sqrt{\omega}}+0.01
$$

For laboratory measurements, the structural coupling losses are not usually as high as in the field. According to Hopkins [16, p. 159], a reasonable indication of the range for the total loss factor of masonry/concrete walls and floors in both laboratory and field conditions is given by

$$
\frac{0.75}{\sqrt{\omega}}+0.01 \leq \eta_{2} \leq \frac{2.5}{\sqrt{\omega}}+0.01
$$

These bounds are in agreement with the measured values for the hollow brick wall, see Table 1 and Fig. 9. In the computations, it will be assumed that the wall's damping loss factor is uniformly distributed in between these bounds. This is in correspondence with the maximum entropy principle, from which it can be derived that when the only information on a random parameter are its bounds, the probability distribution that is consistent with this information is the uniform distribution $[48,49]$.

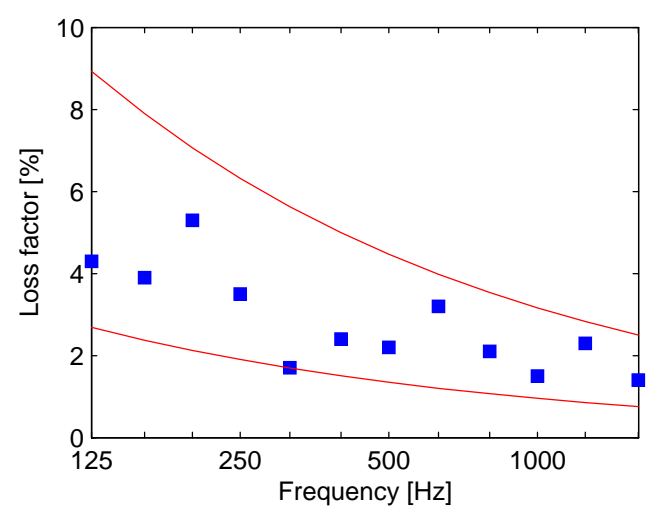

Figure 9: Damping loss factor of a lightweight perforated brick wall. Squares: measured values. Solid lines: bounds according to (28).

For each value of the frequency $\omega$ and wall damping $\eta_{2}$, the hybrid method yields the conditional mean $\mathrm{E}\left[R \mid \eta_{2}\right]$ and the conditional variance $\sigma_{R \mid \eta_{2}}^{2}$ of the transmission loss, accounting for random scattering only: they are given by (23) and (24), respectively. The unconditional statistics, accounting for both random scattering and uncertainty in the damping parameter $\eta_{2}$, can be computed by applying the rules of conditional probability:

$$
\begin{aligned}
\mathrm{E}[R] & =\int_{\eta_{2,1}}^{\eta_{2, \mathrm{u}}} \mathrm{E}\left[R \mid \eta_{2}\right] \frac{1}{\eta_{2, \mathrm{u}}-\eta_{2, \mathrm{l}}} \mathrm{d} \eta_{2} \\
\sigma_{R}^{2} & =\int_{\eta_{2,1}}^{\eta_{2, \mathrm{u}}}\left(\mathrm{E}\left[R \mid \eta_{2}\right]^{2}+\sigma_{R \mid \eta_{2}}^{2}\right) \frac{1}{\eta_{2, \mathrm{u}}-\eta_{2,1}} \mathrm{~d} \eta_{2}-\mathrm{E}[R]^{2},
\end{aligned}
$$

where $\eta_{2,1}$ and $\eta_{2, \mathrm{u}}$ are the lower and upper bounds of $\eta_{2}$ at a particular frequency according to (28). The integrals can be evaluated numerically. The resulting mean and $2 \sigma$ confidence interval are plotted in Fig. $8 \mathrm{~b}$ together with the measured values. By comparing Fig. $8 \mathrm{a}$ and Fig. $8 \mathrm{~b}$, one can observe that, in this application, the additional effect of the uncertainty in the wall's damping loss factor on the uncertainty of the transmission loss is small in the mid-frequency range except that the wall's resonance dips are less pronounced. This can be explained by the fact that, for most frequencies, the measured damping loss factors are relatively close to the lower bound of the assumed probability distribution (see Fig. 9). 


\subsection{Double glazing}

The final structure consists of two glass panes, $6 \mathrm{~mm}$ and $8 \mathrm{~mm}$ thick, separated by an air cavity of $12 \mathrm{~mm}$ (Fig. 5c). The dimensions are $1.20 \mathrm{~m} \times 1.45 \mathrm{~m}$. As material properties of the glass, a density of $\rho=2500 \mathrm{~kg} / \mathrm{m}^{3}$, a modulus of elasticity of $E=62 \mathrm{GPa}$, and a Poisson's ratio of $\nu=0.24$ are taken. For the damping loss factor of the glass panes, measured values are used, while the air cavity is taken to be undamped.

The glazing is modeled with a Rayleigh-Ritz approach, where the analytic modes of the decoupled simply supported glass panes and the decoupled hard-walled cavity are taken as Ritz basis vectors. This deterministic wall model is then coupled to the nonparametric stochastic models of the rooms with the hybrid method, and the sound transmission loss is computed.
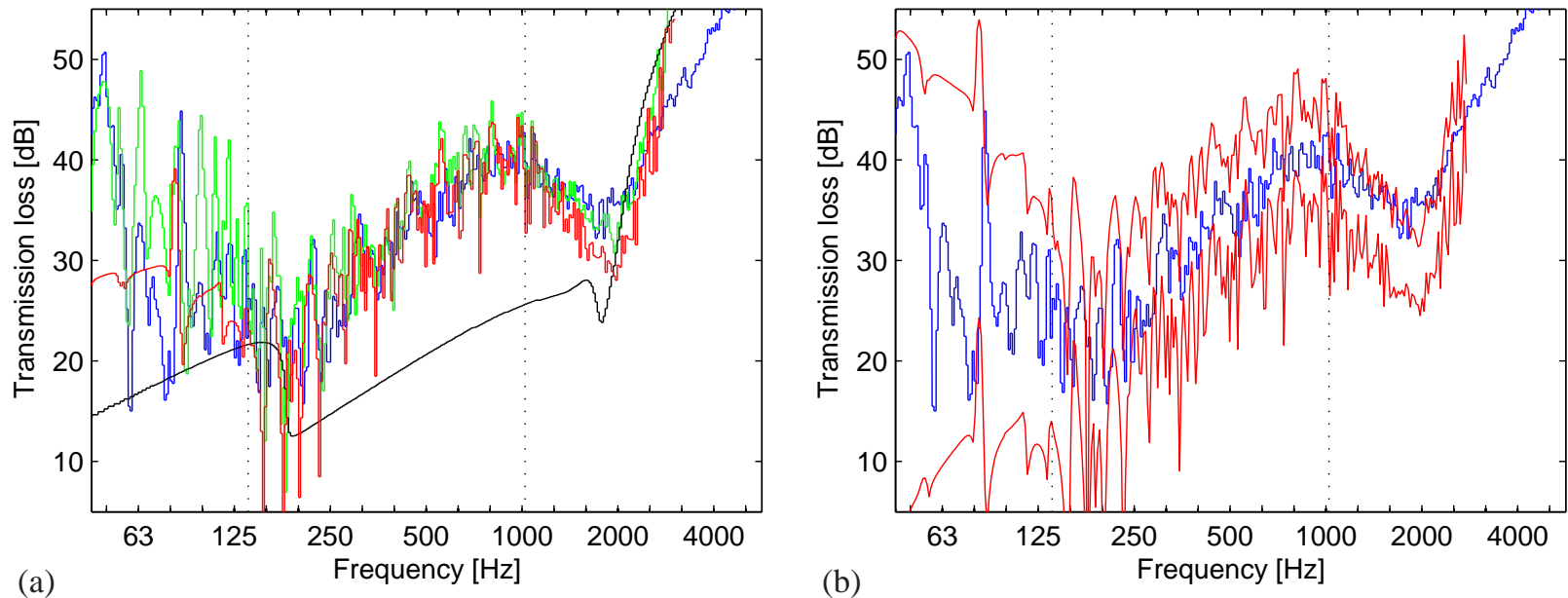

Figure 10: Sound transmission loss of double glazing $6-12-8 \mathrm{~mm}$. (a) Predicted mean, computed with the hybrid method (red), vs. laboratory measurements (blue) and deterministic predictions with the TMM (black) and WBM (green) methods (color online). (b) $2 \sigma$ confidence interval of the hybrid model predictions (red) vs. laboratory measurements (blue) (color online). The considered frequency range is bounded with vertical lines.

The mean of the computed sound transmission loss is plotted in Fig. 10 together with the $2 \sigma$ confidence interval. Individual resonance dips of the glazing are very clearly visible in the hybrid model predictions because of the high stiffness of the glass panes and the low damping (the air cavity was taken to be undamped). A dip in the transmission loss is also visible around $186.5 \mathrm{~Hz}$ which corresponds with the mass-spring-mass resonance frequency of the glass panes on the thin air layer when the plane dimensions of the glazing would be extended towards infinity. Measured values, averaged over 1/48-octave bands, are also shown. An excellent correspondence between the measured and predicted values is observed in the mid-frequency range. The uncertainty caused by random wave scattering is large: the width of the $95 \%$ confidence interval decreases slowly from $21 \mathrm{~dB}$ at $139 \mathrm{~Hz}$ to $10 \mathrm{~dB}$ at $1022 \mathrm{~Hz}$.

In Fig. 10, the measurements and hybrid model predictions are also compared with the predicted values obtained with two deterministic methods in a previous study [50]: the transfer matrix method (TMM) and the wave-based method (WBM). The transfer matrix method [51] models the double glazing as consisting of infinite layers of glass and air and the adjacent rooms as infinite half spaces with diffuse sound field. This method largely underestimates the transmission loss between the mass-spring-mass resonance frequency of the glazing and the coincidence frequency. As discussed in [50], this cannot be resolved by applying correction terms for the diffraction effects by spatially windowing the results [52] or a Gaussian distribution of incident energy [53]. This illustrates that, for double walls, it is important to take the modal behavior of the finite partition structure into account in this broad frequency range. The wave-based method $[54,55]$ is a deterministic method that takes the modal behavior of both the rooms and the glazing into account. It shows a very good agreement with the measured data, but it requires a much larger computational effort than the hybrid method as the rooms need to be modeled with a large number of degrees of freedom (fundamental wave solutions), and it does not provide information on the uncertainty caused by small variations in the acoustic mass and stiffness distributions, which have a wave scattering effect. 


\section{Conclusions}

A stochastic method for sound transmission loss prediction, consisting of a hybridization of displacement-based and energy-based modeling, has been presented. It is especially suited for mid-frequency analysis, and in particular the situation where the wall or floor is modally sparse has been investigated. Compared to deterministic methods such as finite element analysis or the wave-based method, the method is computationally very cheap, since the rooms are modeled in a very efficient, nonparametric stochastic way, as in statistical energy analysis.

The method automatically accounts for uncertainty in spatial variations in geometry, material properties, and boundary conditions, which have a wave scattering effect, in the SEA subsystems. It leads to transmission loss predictions that are robust with respect to such variations: not only mean values, but also variances are obtained. The uncertainty caused by the random wave scatterers is important except when the modal overlap in the rooms is very high. If necessary, additional parametric uncertainty can be accounted for in a straightforward way, as was illustrated for a perforated brick wall with uncertain damping loss factor.

The method was extensively validated against a full modal model of a transmission suite with random acoustic mass distribution, as well as laboratory measurements for wall systems with increasing complexity. In all cases the hybrid method showed an excellent predictive performance within its range of validity.

\section{Appendix A. Transition from low- to high-frequency behavior}

As previously discussed, a vibro-acoustic component can be modeled in an efficient, nonparametric stochastic way (i.e., as carrying a reverberant field) when its natural frequencies are sensitive enough to the small random wave scattering elements that are present. The question remains as to whether the onset of this high-frequency behavior can be estimated. The purpose of this appendix is to offer an approximative method that serves this purpose.

It is known that a given subsystem displays high-frequency behavior when its statistical overlap (not the modal overlap), defined as $S=2 \sigma / \mu$ with $\sigma$ the standard deviation of a natural frequency and $\mu$ the mean frequency spacing, becomes larger than unity, i.e., for $S \geq 1[14,15]$. The following paragraphs indicate how the statistical overlap can be roughly estimated from a modal decomposition of the decoupled unperturbed subsystem, by generalizing the analysis of [56] for plate structures to arbitrary structural and acoustic subsystems. Only the (acoustic) mass effect of the random wave scatterers are accounted for, as it normally dominates the statistical overlap at higher frequencies. If necessary the expressions can be extended so as to include uncertainty of the stiffness distribution.

Structural components. An idealized way of modeling the mass effect of the random wave scatterers is by considering them as (acoustic) point masses. In the following analysis, a total of $N_{\mathrm{m}}$ point masses are attached to the component at random locations. All point masses have the same magnitude $\delta m$. For an undamped structural component, the change of the natural frequency of mode $j$ to the set of random local mass changes is up to first-order accuracy given by [57]

$$
\delta \omega_{j}=-\frac{\omega_{j}}{2} \sum_{k=1}^{N_{\mathrm{m}}} \varphi_{j}^{2}\left(\mathbf{x}_{k}\right) \delta m,
$$

where $\omega_{j}$ is the natural frequency of mode $j$ in $\mathrm{rad} / \mathrm{s}$ and $\varphi_{j}\left(\mathbf{x}_{k}\right)$ is the mass-normalized mode shape of mode $j$ evaluated at the random position $\mathbf{x}_{k}$. Since the mode shapes are mass-normalized, one has that

$$
\iiint \rho \varphi_{j}^{2}(\mathbf{x}) \mathrm{d} x \mathrm{~d} y \mathrm{~d} z=1
$$

where $\rho$ denotes the density. Taking the expected value and assuming that the variance is independent from the position yields

$$
\mathrm{E}\left[\varphi_{j}^{2}\left(\mathbf{x}_{k}\right)\right]=\frac{1}{M}
$$

with $M$ the total mass of the substructure. Substitution into (A.1) results in the following expression for the expected value of the natural frequency change:

$$
\mathrm{E}\left[\delta \omega_{j}\right]=-\frac{\omega_{j} N_{\mathrm{m}} \delta m}{2 M}
$$


Taking the square and subsequently the expected value of both sides of (A.1) yields

$$
\mathrm{E}\left[\left(\delta \omega_{j}\right)^{2}\right]=\frac{\omega_{j}^{2}(\delta m)^{2}}{4} \sum_{k=1}^{N_{\mathrm{m}}} \sum_{l=1}^{N_{\mathrm{m}}} \mathrm{E}\left[\varphi_{j}^{2}\left(\mathbf{x}_{k}\right) \varphi_{j}^{2}\left(\mathbf{x}_{l}\right)\right] .
$$

By assuming that the mode shapes are statistically independent at the various points where the masses are attached, one has that

$$
\mathrm{E}\left[\left(\delta \omega_{j}\right)^{2}\right]=\frac{\omega_{j}^{2}(\delta m)^{2}}{4}\left(N_{\mathrm{m}} \mathrm{E}\left[\varphi_{j}^{4}\left(\mathbf{x}_{k}\right)\right]+N_{\mathrm{m}}\left(N_{\mathrm{m}}-1\right) \mathrm{E}\left[\varphi_{j}^{2}\left(\mathbf{x}_{k}\right)\right]^{2}\right) .
$$

If the mode shapes of the component are sinusoidal ${ }^{1}$, then their second and fourth moment are related by [17, p. 101]:

$$
\mathrm{E}\left[\varphi_{j}^{4}\left(\mathbf{x}_{k}\right)\right]=\left(\frac{3}{2}\right)^{D} \mathrm{E}\left[\varphi_{j}^{2}\left(\mathbf{x}_{k}\right)\right]^{2}
$$

where $D$ denotes the dimensionality of the component, i.e., $D=1$ for a beam, $D=2$ for a plate and $D=3$ for a volume. The dimensionality of a specific component is of course frequency dependent: it may be defined as the number of dimensions that are large compared to the wavelength at that frequency. Substitution into (A.6) and applying (A.3) yields

$$
\mathrm{E}\left[\left(\delta \omega_{j}\right)^{2}\right]=\frac{\omega_{j}^{2}(\delta m)^{2} N_{\mathrm{m}}}{4 M^{2}}\left(\left(\frac{3}{2}\right)^{D}+N_{\mathrm{m}}-1\right) .
$$

Given (A.4) and (A.8), the variance of the natural frequency of mode $j$ is obtained as

$$
\begin{aligned}
\sigma^{2} & =\mathrm{E}\left[\left(\delta \omega_{j}\right)^{2}\right]-\mathrm{E}\left[\delta \omega_{j}\right]^{2} \\
& =\frac{\omega_{j}^{2}(\delta m)^{2} N_{\mathrm{m}}}{4 M^{2}}\left(\left(\frac{3}{2}\right)^{D}-1\right) .
\end{aligned}
$$

With this result, the statistical overlap becomes

$$
S=\frac{\omega_{j} n \delta m}{M} \sqrt{N_{\mathrm{m}}\left(\left(\frac{3}{2}\right)^{D}-1\right)}
$$

where $n$ denotes the modal density in rad/s. For the special case of a thin plate, all quantities in (A.11) are fairly constant with frequency except $\omega_{j}$, and $S$ approximately increases linearly with frequency, as shown in Fig. A.11a. For thick plates, the modal density is constant at lower frequencies but it increases linearly with frequency when the shear stiffness starts to play an important role [58]. In this case, the statistical overlap increases first linearly and then quadratically with frequency, see Fig. A.11b.

Acoustic components. Adding a point mass to a structural component without changing its stiffness essentially increases its density in a volume that is so small that the variation of the displacement within that volume is negligible. The acoustic equivalent is creating a 'point air pocket' by decreasing the bulk modulus (hence the sound speed) in a volume that is so small that the variation of the pressure within that volume is negligible. Denoting the volume and sound speed in a point air pocket at location $k$ by $V_{k}$ and $c_{k}$, respectively, the corresponding acoustic point mass is $m_{k}=V_{k} / c_{k}^{2}$. Following the same lines as for structural components, the statistical overlap of an acoustic component due to local acoustic mass changes of amplitude $\delta m$ at $N_{\mathrm{m}}$ random locations is

$$
S=\frac{\omega_{j} n V \delta m}{c^{2}} \sqrt{N_{\mathrm{m}}\left(\left(\frac{3}{2}\right)^{D}-1\right)}
$$

\footnotetext{
${ }^{1}$ Note that, according to diffuse field theory, the mode shapes of an SEA subsystem are Gaussian random fields when viewed across the random ensemble. However, in the present derivation the mode shapes of the deterministic, unperturbed component are considered. They are taken to be sinusoidal, and the location at which they are evaluated is randomly varied.
} 

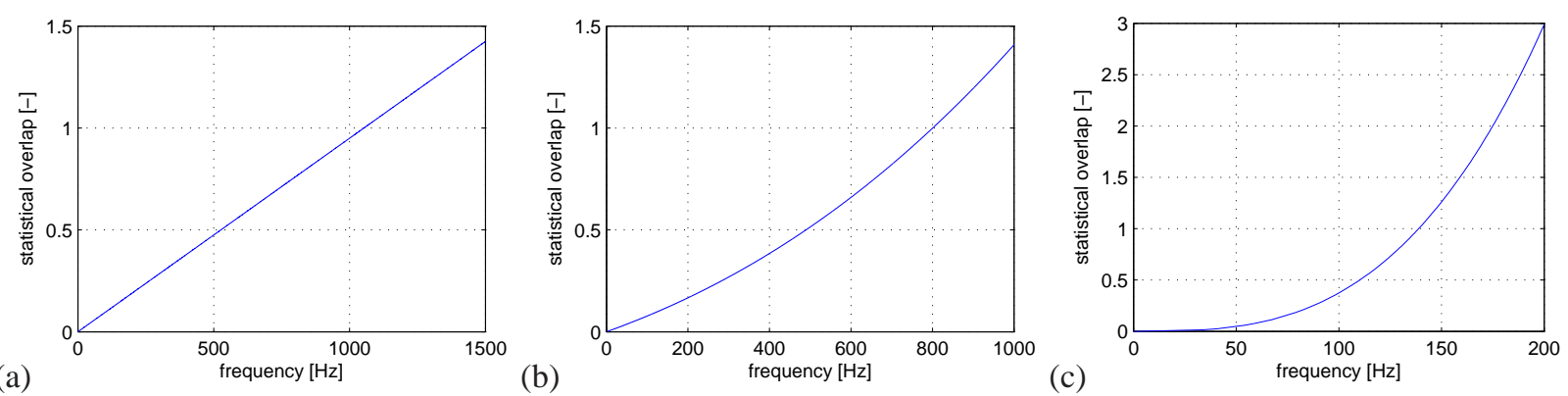

Figure A.11: Estimated statistical overlap of (a) the bending modes of the thin bare PMMA plate, (b) the combined bending-shear modes of the bare perforated brick wall and (c) the acoustic modes of the reverberation room, detailed in section 3 .

where $c$ denotes the nominal sound speed and $V$ is the total volume of the acoustic cavity. Again, $D$ denotes the dimensionality of the component, i.e., $D=1$ for a long tube, $D=2$ for a thin air layer and $D=3$ for a room. Of course the dimensionality is frequency dependent: it may be defined as the number of dimensions that are large compared to the wavelength at that frequency. In a room, the modal density increases quadratically with frequency, so $S$ grows cubically with frequency (Fig. A.11c).

Examples and discussion. Equations (A.11) and (A.12) each contain two parameters to be estimated by the analyst: the number of (acoustic) masses $N_{\mathrm{m}}$ and the associated individual mass $\delta m$. In the simulation example of section 2.5, the number of acoustic masses $N_{\mathrm{m}}$ and their magnitude $\delta m$ are known. The statistical overlap of the rooms can therefore be directly computed from (A.12) with $D=3$. The value $S=1$ is obtained at $139 \mathrm{~Hz}$. As can be seen from Figs. 3 and 4, the response statistics predicted by a hybrid model, where the wall is deterministic and the rooms are SEA subsystems, accurately reflect the true response statistics for $S \geq 1$.

In the experimental case studies of section $3, N_{\mathrm{m}}$ and $\delta m$ are estimated as follows. First, a rough estimate is made of which portion of the total (acoustic) mass of a vibro-acoustic component is random. A portion of $4 \%$ is nominally assumed in the applications except for the perforated brick wall, where the presence of the perforations clearly causes a higher mass uncertainty, roughly estimated at $20 \%$. Then, an estimate of the number of random wave scatterers is made. A value $N_{\mathrm{m}}=20$ is nominally assumed in the applications, except for the brick wall where the perforations are clearly more homogenously distributed, and $N_{\mathrm{m}}=100$ is taken. The corresponding transition frequencies are: $139 \mathrm{~Hz}$ for the rooms, $1053 \mathrm{~Hz}$ for the PMMA panel of section 3.1, $1149 \mathrm{~Hz}$ for the gypsum block wall of section 3.2, $801 \mathrm{~Hz}$ for the perforated brick wall of section 3.3, and $1022 \mathrm{~Hz}$ for the $6 \mathrm{~mm}$ glazing of section 3.4.

The question remains as to how sensitive the estimated transition frequency is to these rough estimates of $N_{\mathrm{m}}$ and $\delta m$. From (A.11) and (A.12), it is clear that the statistical overlap is proportional to $\sqrt{N_{\mathrm{m}}} \delta m$. For thin plates in bending, the statistical overlap scales linearly with frequency, so the estimated transition frequency is also proportional to $\sqrt{N_{\mathrm{m}}} \delta m$. However, for acoustic room volumes, the statistical overlap scales cubically with frequency and therefore the estimated transition frequency is only proportional to $\sqrt[6]{N_{\mathrm{m}}(\delta m)^{2}}$.

This implies that the transition frequency for plates is more sensitive to the estimated values of $N_{\mathrm{m}}$ and $\delta m$ than the transition frequency for rooms. Consider the subsystems of Fig. A.11 as an example. When the number of point masses of the PMMA plate (Fig. A.11a) is doubled from 20 to 40 and $\delta m$ is kept constant, the transition frequency of the plate decreases from $1053 \mathrm{~Hz}$ to $745 \mathrm{~Hz}$. When the number of acoustic point masses in a room (Fig. A.11c) is doubled from 20 to 40 and $\delta m$ is kept constant, the transition frequency of the room decreases only slightly, from $139 \mathrm{~Hz}$ to $124 \mathrm{~Hz}$.

\section{Acknowledgements}

This research was funded by the Research Foundation - Flanders (FWO), Belgium, under a Postdoctoral Research Fellowship provided to Edwin Reynders. 


\section{References}

[1] T. Kihlman, A. Nilsson, The effects of some laboratory designs and mounting conditions on reduction index measurements, Journal of Sound and Vibration 24 (3) (1972) 349-364.

[2] M. Bhattacharya, R. Guy, The influence of the measuring facility on the measured sound insulating property of a panel, Acustica 26 (1972) $344-348$.

[3] R. Guy, A. De Mey, P. Sauer, The effect of some physical parameters upon the laboratory measurements of sound transmission loss, Applied Acoustics 18 (2) (1985) 81-98.

[4] A. Osipov, P. Mees, G. Vermeir, Low-frequency airborne sound transmission through single partitions in buildings, Applied Acoustics 52 (34) (1997) 273-288.

[5] D. Pedersen, J. Roland, G. Raabe, W. Maysenhölder, Measurement of the low-frequency sound insulation of building components, Acta Acustica united with Acustica 86 (3) (2000) 495-505.

[6] A. Dijckmans, G. Vermeir, Numerical investigation of the repeatability and reproducibility of laboratory sound insulation measurements, Acta Acustica united with Acustica 99 (3) (2013) 421-432.

[7] E. Reynders, Parametric uncertainty quantification of sound insulation values, Journal of the Acoustical Society of America 135 (4) (2014) 1907-1918.

[8] K. A. Mulholland, R. H. Lyon, Sound insulation at low frequencies, Journal of the Acoustical Society of America 54 (4) (1973) $867-878$.

[9] L. Gagliardini, J. Roland, J. Guyader, The use of a functional basis to calculate acoustic transmission between rooms, Journal of Sound and Vibration 145 (3) (1991) 457-478.

[10] S. Maluski, B. Gibbs, Application of a finite-element model to low-frequency sound insulation in dwellings, Journal of the Acoustical Society of America 108 (4) (2000) 1741-1751.

[11] A. Dijckmans, G. Vermeir, Development of a hybrid wave based - transfer matrix model for sound transmission analysis, Journal of the Acoustical Society of America 133 (4) (2013) 2157-2168.

[12] A. Deraemaeker, I. Babuška, P. Bouillard, Dispersion and pollution of the FEM solution for the Helmholtz equation in one, two and three dimensions, International Journal for Numerical Methods in Engineering 46 (4) (1999) 471-499.

[13] R. Bernhard, The limits of predictability due to manufacturing and environmentally induced uncertainty, in: Proceedings of Inter-Noise 96, the 25th International Congress on Noise Control Engineering, Liverpool, U.K., 1996.

[14] C. Manohar, A. Keane, Statistics of energy flows in spring-coupled one-dimensional subsystems, Philosophical Transactions of the Royal Society A - Mathematical, Physical and Engineering Sciences 346 (1681) (1994) 525-542.

[15] R. Langley, On the diffuse field reciprocity relationship and vibrational energy variance in a random subsystem at high frequencies, Journal of the Acoustical Society of America 121 (2) (2007) 913-921.

[16] C. Hopkins, Sound insulation, Elsevier Ltd., Oxford, 2007.

[17] R. Lyon, R. DeJong, Theory and application of statistical energy analysis, 2nd Edition, Butterworth-Heinemann, Newton, MA, 1995.

[18] R. Craik, Sound transmission through buildings using statistical energy analysis, Gower, Aldershot, UK, 1996.

[19] L. Cremer, M. Heckl, B. Petersson, Structure-borne sound: Structural vibrations and sound radiation at audio frequencies, 3rd Edition, Springer, Berlin, 2005.

[20] R. Weaver, On the ensemble variance of reverberation room transmission functions, the effect of spectral rigidity, Journal of Sound and Vibration 130 (3) (1989) 487-491.

[21] M. Mehta, Random Matrices, 3rd Edition, Elsevier, San Diego, CA, 2004.

[22] R. Lyon, Statistical analysis of power injection and response in structures and rooms, Journal of the Acoustical Society of America 45 (3) (1969) 545-565.

[23] J. Davy, The relative variance of the transmission function of a reverberation room, Journal of Sound and Vibration 77 (4) (1981) $455-479$.

[24] R. Langley, A. Brown, The ensemble statistics of the energy of a random system subjected to harmonic excitation, Journal of Sound and Vibration 275 (3-5) (2004) 823-846.

[25] R. Langley, A. Brown, The ensemble statistics of the band-averaged energy of a random system, Journal of Sound and Vibration 275 (3-5) (2004) 847-857.

[26] R. Langley, V. Cotoni, Response variance prediction in the statistical energy analysis of built-up systems, Journal of the Acoustical Society of America 115 (2) (2004) 706-718.

[27] V. Cotoni, R. Langley, M. Kidner, Numerical and experimental validation of variance prediction in the statistical energy analysis of built-up systems, Journal of Sound and Vibration 288 (3) (2005) 701-728.

[28] E. Reynders, R. Langley, Response probability distribution of built-up vibro-acoustic systems, Journal of the Acoustical Society of America 131 (2) (2012) 1138-1149.

[29] R. Langley, J. Legault, J. Woodhouse, E. Reynders, On the applicability of the lognormal distribution in random dynamical systems, Journal of Sound and Vibration 332 (13) (2013) 3289-3302.

[30] P. Shorter, R. Langley, On the reciprocity relationship between direct field radiation and diffuse reverberant loading, Journal of the Acoustical Society of America 117 (1) (2005) 85-95.

[31] P. Shorter, R. Langley, Vibro-acoustic analysis of complex systems, Journal of Sound and Vibration 288 (3) (2005) 669-699.

[32] R. Langley, V. Cotoni, Response variance prediction for uncertain vibro-acoustic systems using a hybrid deterministic-statistical method, Journal of the Acoustical Society of America 122 (6) (2007) 3445-3463.

[33] A. Cicirello, R. Langley, The vibro-acoustic analysis of built-up systems using a hybrid method with parametric and non-parametric uncertainties, Journal of Sound and Vibration 332 (9) (2013) 2165-2178.

[34] C. Díaz-Cereceda, J. Poblet-Puig, A. Rodríguez-Ferran, The finite layer method for modelling the sound transmission through double walls, Journal of Sound and Vibration 331 (22) (2012) 4884-4900.

[35] C. Huang, S. Nutt, An analytical study of sound transmission through unbounded panels of functionally graded materials, Journal of Sound and Vibration 330 (6) (2011) 1153-1165. 
[36] J. Legault, A. Mejdi, N. Atalla, Vibro-acoustic response of orthogonally stiffened panels: the effects of finite dimensions, Journal of Sound and Vibration 330 (24) (2011) 5928-5948

[37] A. Mejdi, J. Legault, N. Atalla, Transmission loss of periodically stiffened laminate composite panels: shear deformation and in-plane interaction effects, Journal of the Acoustical Society of America 131 (1) (2012) 174-185.

[38] F. Xin, T. Lu, C. Chen, Sound transmission through simply supported finite double-panel partitions with enclosed air cavity, ASME Journal of Vibration and Acoustics 132 (1) (2010) 011008

[39] R. Langley, J. Cordioli, Hybrid deterministic-statistic analysis of vibro-acoustic systems with domain couplings on statistical components, Journal of Sound and Vibration 321 (3-5) (2009) 893-912.

[40] E. Williams, G. Maynard, Numerical evaluation of the Rayleigh integral for planar radiators using the FFT, Journal of the Acoustical Society of America 72 (6) (1982) 2020-2030.

[41] R. Langley, Numerical evaluation of the acoustic radiation from planar structures with general baffle conditions using wavelets, Journal of the Acoustical Society of America 121 (2) (2007) 766-777.

[42] International Organization for Standardization, ISO 10140-2:2010: Acoustics - Laboratory measurement of sound insulation of building elements - Part 2: Measurement of airborne sound insulation (2010).

[43] F. Fahy, P. Gardonio, Sound and structural vibration, 2nd Edition, Academic Press, Oxford, UK, 2007.

[44] C. Jeyachandrabose, J. Kirkhope, Construction of new efficient three-node triangular thin plate bending elements, Computers and Structures 23 (5) (1986) 587-603.

[45] International Organization for Standardization, ISO 10140:2010: Acoustics - Laboratory measurement of sound insulation of building elements (2010)

[46] G. Jacqus, S. Berger, V. Gibiat, P. Jean, M. Villot, S. Ciukay, A homogenised vibratory model for predicting the acoustic properties of hollow brick walls, Journal of Sound and Vibration 330 (14) (2011) 3400-3409.

[47] R. Craik, Damping of building structures, Applied Acoustics 14 (5) (1981) 347-359.

[48] E. Jaynes, Information theory and statistical mechanics, The Physical Review 106 (4) (1957) 620-630.

[49] E. Jaynes, Probability Theory. The Logic of Science, Cambridge University Press, Cambridge, UK, 2003.

[50] A. Dijckmans, G. Vermeir, W. Lauriks, Sound transmission through finite lightweight multilayered structures with thin air layers, Journal of the Acoustical Society of America 128 (6) (2010) 3513-3524.

[51] W. Lauriks, P. Mees, J. F. Allard, The acoustic transmission through layered systems, Journal of Sound and Vibration 155 (1) (1992) $125-132$.

[52] M. Villot, C. Guigou, L. Gagliardini, Predicting the acoustical radiation of finite size multi-layered structures by applying spatial windowing on infinite structures, Journal of Sound and Vibration 245 (3) (2001) 433-455.

[53] H. Kang, J. Ih, J. Kim, H. Kim, Prediction of sound transmission loss through multilayered panels by using gaussian distribution of directional incident energy, Journal of the Acoustical Society of America 107 (3) (2000) 1413-1420.

[54] W. Desmet, B. van Hal, P. Sas, D. Vandepitte, A computationally efficient prediction technique for the steady-state dynamic analysis of coupled vibro-acoustic systems, Advances in Engineering Software 33 (2002) 527-540.

[55] B. Pluymers, B. van Hal, D. Vandepitte, W. Desmet, Trefftz-based methods for time-harmonic acoustics, Archives of Computational Methods in Engineering 14 (4) (2007) 343-381.

[56] N. Kessissoglou, G. Lucas, Gaussian orthogonal ensemble spacing statistics and the statistical overlap factor applied to dynamic systems, Journal of Sound and Vibration 324 (3-5) (2009) 1039-1066.

[57] R. Fox, M. Kapoor, Rate of change of eigenvalues and eigenvectors, AIAA Journal 6 (12) (1968) 2426-2429.

[58] J. Rindel, Dispersion and absorption of structure-borne sound in acoustically thick plates, Journal of Sound and Vibration 41 (2) (1994) $97-111$. 